Monatsschr Kinderheilkd 2018 · 166:611-612 https://doi.org/10.1007/s00112-018-0529-8

Online publiziert: 11. Juni 2018

(c) Springer Medizin Verlag $\mathrm{GmbH}$, ein Teil von Springer Nature 2018

CrossMark

P. Weber $\cdot$ R. John ${ }^{2} \cdot$ K. Konrad ${ }^{3} \cdot$ B. v. Livonius ${ }^{4} \cdot$ B. Lorenz ${ }^{5} \cdot$ B. Ruple ${ }^{6} \cdot$ S. StockMühlnickel $^{7} \cdot$ D. Karch ${ }^{8} \cdot$ A. Schroeder ${ }^{9}$

${ }^{1}$ Abt. Neuro- und Entwicklungspädiatrie, Universitäts-Kinderspital beider Basel, Basel, Schweiz

${ }^{2}$ Campus Virchow, SPZ Charité, Berlin, Deutschland

${ }^{3}$ Lehr- und Forschungsgebietes Klinische Neuropsychologie des Kindes- und Jugendalters, Universitätsklinikum der RWTH Aachen, Aachen, Deutschland

${ }^{4}$ Sektion Vergrößernde Sehhilfen; Augenklinik der LMU, Klinikum der Universität München, München, Deutschland

${ }^{5}$ Klinik und Poliklinik für Augenheilkunde, Universitätsklinikum Gießen, Gießen, Deutschland

${ }^{6}$ Nikolauspflege Stiftung für blinde und sehbehinderte Menschen, Stuttgart, Deutschland

${ }^{7}$ Abteilung Ergotherapie, Universitäts-Kinderspital beider Basel, Basel, Schweiz

${ }^{8}$ Klinik für Kinderneurologie und Sozialpädiatrie, Kinderzentrum Maulbronn gGmbH, Maulbronn, Deutschland

${ }^{9}$ Werner Otto Institut gGmbH, Hamburg, Deutschland

\title{
Erratum zu: Visuelle Wahrnehmungsstörungen
}

\section{Zusammenfassung der Sk2-Leitlinie AWMF- Registernummer 022/020}

\section{Erratum zu:}

Monatsschr Kinderheilkd 2018

https://doi.org/10.1007/s00112-018-

0462-x

Im Literaturverzeichnis [4] wird fälschlich auf die AWMF-Leitlinie Autismusspektrum-Störung (028-018) verwiesen (http://www.awmf.org/uploads/ tx_szleitlinien/028-0181_S3_AutismusSpektrum-Stoerungen_ASS-Diagnos tik_2016-05.pdf).

Bitte beachten Sie den hier aufgeführten richtigen Link auf die Sk2-Leitlinie „Visuelle Wahrnehmungsstörungen (AWMF-Registernummer 022/020): http://www.awmf.org/uploads/tx_szleit linien/022-0201_S2k_Visuelle-Wahrneh mungsstoerungen_2017-12.pdf.

Wir bitten diesen Fehler zu entschuldigen und die korrekte Literaturangabe zu berücksichtigen.

\section{Korrespondenzadresse}

Prof. Dr. med. Dipl.-Psych. P. Weber Abt. Neuro- und Entwicklungspädiatrie, Universitäts-Kinderspital beider Basel Basel, Schweiz

peter.weber@ukbb.ch

\section{Literatur}

4. AWMF (2017) Sk2-Leitlinie „Visuelle Wahrnehmungsstörungen" (AWMF-Registernummer 022/020). http://www.awmf.org/ uploads/tx_szleitlinien/022-020l_S2k_VisuelleWahrnehmungsstoerungen_2017-12.pdf.Zugegriffen: 30.04 .2017 
Hier steht eine Anzeige.

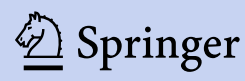

